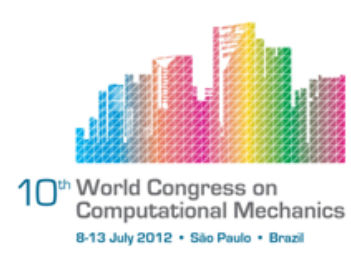

\title{
THE NUMERICAL SIMULATION OF LARGE-SCALE FLUID-STRUCTURE INTERACTION PROBLEMS IN A FULLY COUPLED FORM
}

\author{
A. Eken $^{1}$, M. Sahin ${ }^{2}$ \\ ${ }^{1}$ Aeronautical Engineering Department, Istanbul Technical University \\ ${ }^{2}$ Astronautical Engineering Department, Istanbul Technical University \\ (msahin.ae00@gtalumni.org)
}

\begin{abstract}
A fully coupled numerical algorithm has been developed for the numerical simulation of large-scale fluid structure interaction problems. The incompressible Navier-Stokes equations are discretized using an Arbitrary Lagrangian-Eulerian (ALE) formulation based on the side-centered unstructured finite volume method. The present arrangement of the primitive variables leads to a stable numerical scheme and it does not require any ad-hoc modifications in order to enhance the pressure-velocity coupling. A special attention is given to satisfy the discrete continuity equation within each element at discrete level as well as the Geometric Conservation Law (GCL). The nonlinear elasticity equations are discretized within the structure domain using the Galerkin finite element method. The resulting algebraic linear equations are solved in a fully coupled form. The implementation of the fully coupled preconditioned iterative solvers is based on the PETSc library for improving th! e efficiency of the parallel code. The present numerical algorithm is initially validated for a Newtonian fluid interacting with an elastic rectangular bar behind a circular cylinder and a three-dimensional elastic solid confined in a rectangular channel.

ical Galerkin finite element is used to discretize the governing equations in a Lagrangian frame. The time integration method for the structure domain is based on the Newmark type generalized $-\alpha$ method while the first-order backward difference is used in the fluid domain. The implementation of the preconditioned coupled iterative solvers is based on the PETSc library for improving the efficiency of the parallel code. The present numerical algorithm is validated for a steady and unsteady Newtonian fluid interacting with an elastic rectangular bar behind a circular cylinder and a three-dimensional elastic solid confined in a rectangular channel.
\end{abstract}

Keywords: Fluid-Structure Interaction, Unstructured Finite Volume Method, Finite Element Method, Large Displacement, Large-Scale Computation; Monolitic Method.

\section{INTRODUCTION}

Fluid-structure interactions (FSI), that is interactions of some movable or deformable structure with an internal or surrounding fluid flow, are of great importance due to their many 
engineering and biomedical applications. The most common applications to problems in engineering include wing flutter, tail buffeting, flow induced vibrations in heat exchangers tubes, large class of acoustic problems, fluid-structure-combustion interactions occurring within the combustion chambers, interaction process in rocket nozzles, fuel tank sloshing, wave structure interactions, explosions or high-velocity impacts, underwater implosions, structural effect of strong wind on bridges and tall buildings, etc. On the other hand, much of the work in biomedical applications include animal locomotion, blood flow in the cardiovascular system and dynamics of heart valves. Accurate prediction of fluid-structure interactions is crucial for many engineering structures in order to avoid potential aeroe! lastic/hydroelastic instability issues. Failing to consider these effects can be catastrophic, especially in structures comprising materials susceptible to fatigue. For example, wing flutter occurs as a result of exchange of energy between different modes of the structure because of fluid-structure interactions and a growing oscillation of a wing surface leading to large amplitudes and stresses, and which can tear an airframe apart.

One of the most well-known methods used to capture the interaction between structure and fluid is the Arbitrary Lagrangian Eulerian (ALE) method as described in Hirt el al. [21]. In the ALE method, the mesh follows the interface between the fluid and solid boundary and the governing equations are discretized on a moving mesh. This differs from the standard Eulerian formulation in a way that the mesh movement has to fulfill special conditions in order to maintain the accuracy and the stability of the time integration scheme. This condition is satisfied by the enforcement of the so-called geometric conservation law (GCL) as coined by Thomas and Lombard [35]. The ALE approach was subsequently adopted within the finite element context to solve free surface problems of incompressible viscous fluid flow [23]. In the case of an FSI problem, the deformable fluid-structure interface is taken into account and the fluid points at the fluid-solid in! terface are moved in a Lagrangian way [13]. The current article presents a new numerical algorithm based on the ALE formulation for a fully coupled solution of the large-scale FSI problems where the fluid is modelled by the incompressible Navier-Stokes equations and the structure is modeled by the St. Venant-Kirchhoff model. The governing equations of the fluid domain are discretized using an Arbitrary LagrangianEulerian (ALE) formulation based on the side-centered unstructured finite volume method where the velocity vector components are defined at the mid-point of each cell face while the pressure is defined at the element centroid. The present arrangement of the primitive variables leads to a stable numerical scheme and it does not require any ad-hoc modifications in order to enhance the pressure-velocity coupling. This approach was initially used by Hwang [24] and Rida et al. [31] for the solution of the incompressible N! avier-Stokes equations on unstructured triangular meshes. The most app ealing feature of the present finite volume approach is that it leads to the classical five-point Laplace operator for the pressure Poisson equation as in the classical MAC scheme [20] which is very important for the efficient solution of the large-scale FSI problems. Because, numerical simulations of FSI problems in general require large computational resources, and it is typically the fluid subproblem that requires the most computational resources in the coupled system [14]. In the present work, a special attention will be given to satisfy the continuity equation exactly within each element and the summation of the continuity equations can be exactly reduced to the domain boundary, which is important for the global mass conservation. The mesh deformation within the fluid domain is achieved by using an algebraic approach based on the minimum distance function at each time level while avoiding remeshing in order to enhance numerical robustness. The deformation of the solid domain is governed by the constitutive laws for the nonlinear Saint Venant-Kirchhoff material and the classical Galerkin finite element method is used to discretize the governing 
equations in a Lagrangian frame. Newmark [28] type generalized- $\alpha$ metho! $\mathrm{d}$ [9] is employed to integrate in time the solid dynamic equilibrium equation.

Numerical methods for the solution of FSI problems lead to highly nonlinear illconditioned systems and the development of robust and efficient solution techniques for such systems presents one of the great challenges in computational mechanics. The computational methods for FSI problems can be classified as partitioned (segregated) [29] or fully coupled (monolithic) [8, 15] approaches. Partitioned methods utilize separate solvers for the fluid and solid domains and attempt to obtain a coupled solution via a fixed-point (Picard) iteration. This approach gives more freedom in selecting suitable methods for fluid and structural solvers than the monolithic approaches. However, partitioned methods also have serious drawbacks. Fixed-point iterations tend to converge slowly and the iterations may diverge in the presence of strong fluid-solid interactions where the ratio of fluid density to solid density is close to one. In addition, partitioned methods can! not satisfy the incompressibility constraint of fluid during standard alternating FSI iterations where the fluid domain is entirely enclosed by Dirichlet boundary conditions. In a fully coupled (monolithic) approach, the fluid and solid equations are discretized and solved simultaneously as a single equation system for the entire problem. However, the solution of a large system of coupled nonlinear algebraic equations is required. It is widely believed, however, that monolithic solvers are more robust than their segregated counterparts, but are believed to be too expensive for use in large-scale problems. Heil et al. [16] demonstrated that monolithic solvers are competitive even in test cases with very weak FSI. In this context, Behr and Tezduyar [6] presented solution strategies for large-scale flow simulations and Johnson and Tezduyar [25] proposed mesh update strategies in parallel computations. Gee et al. [15] applied an algebraic multigrid technique to the entire fluidstructure interaction system of equations. Barker and Cai [3] developed a scalable parallel finite element solver for the si! mulation of blood flow in compliant arteries using scalable Newton-Krylov algorithms preconditioned with an overlapping restricted additive Schwarz method. The current paper also deals with development of efficient preconditioning technique that allows the rapid iterative solution of the resulting large-scale linear systems. The present one level iterative solver is based on the multiplication of the original system with an upper triangular right preconditioner which results in a scaled discrete Laplacian instead of a zero block in the original system. Then the restricted additive Schwarz method with the flexible GMRES $(m)$ algorithm [32] is employed and an incomplete ILU $(k)$ algorithm is used witin each partitioned sub-domains. The two level iterative solver based on the multiplicative non-nested multigrid method with one V-cycle is described in [33] in detail and it has been successfully applied to the solution of the Stokes problem. The implementation of the preconditioned coupled iterative solvers is based on the PETSc library for improving the efficiency of the parallel code. The computational domain is decomposed into a set of sub-domains or partitions using the METIS library [27].

The remainder of this paper is organized as follow: Section 2 provides some detail on the present FSI method with the iterative solvers. In Section 3 the method is initially validated for an nsteady Newtonian fluid interacting with an elastic rectangular bar behind a circular cylinder and a three-dimensional elastic solid confined in a rectangular channel. Concluding remarks are provided in Section 4. 


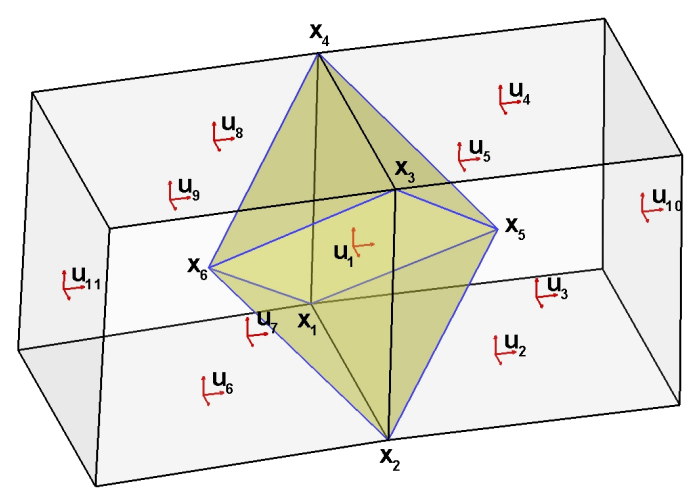

Figure 1. Three-dimensional unstructured mesh with a dual control volume for the velocity components.

\section{MATHEMATICAL and NUMERICAL FORMULATION}

\subsection{Fluid model}

The integral form of the incompressible NavierStokes equations that govern the motion of an arbitrary moving control volume $\Omega(t)$ with boundary $\partial \Omega(t)$ can be written in the Cartesian coordinate system in dimensionless form as follows: the momentum equations

$$
R e \int_{\Omega_{d}} \frac{\partial \mathbf{u}}{\partial t} d V+R e \oint_{\partial \Omega_{d}}[\mathbf{n} \cdot(\mathbf{u}-\dot{\mathbf{x}})] \mathbf{u} d S+\oint_{\partial \Omega_{d}} \mathbf{n} p d S=\oint_{\partial \Omega_{d}} \mathbf{n} \cdot \nabla \mathbf{u} d S
$$

the continuity equation

$$
-\oint_{\partial \Omega_{e}} \mathbf{n} \cdot \mathbf{u} d S=0
$$

In these equations, $V$ is the control volume, $S$ is the control volume surface area, $\mathbf{n}$ represents the outward normal vector, $\mathbf{u}$ represents the local fluid velocity vector, $\dot{\mathbf{x}}$ represents the grid velocity (the velocity vector of the control volume surface), $p$ is the pressure and $R e$ is the dimensionless Reynolds number. Figure 1 illustrates typical two neighboring hexahedral elements with a dual finite volume constructed by connecting the element centroids to the common vertices shared by the both hexahedral elements. The local fluid velocity vector components are defined at the mid-point of each face.

\subsection{Solid model}

The structural behaviour of solid domain is governed by the following conservation of momentum in the Lagrangian framework where the material derivative becomes a partial derivative with respect to time

$$
\rho \frac{\partial^{2} \mathbf{d}}{\partial \mathrm{t}^{2}}=\nabla \cdot \boldsymbol{\sigma}_{s}+\rho \mathbf{b}
$$

where $\rho$ is the solid material density, $\mathbf{d}$ is the displacement vector, $\sigma_{s}$ is the Cauchy stress tensor and $\mathbf{b}$ is the body forces. The material is defined by the Cauchy stress tensor using the 
following constitutive law for the St. Venant-Kirchhoff material:

$$
\begin{aligned}
\mathbf{S} & =J \mathbf{F}^{-1} \sigma_{s} \mathbf{F}^{-\top} \\
\mathbf{F} & =(\mathbf{I}+\nabla \mathbf{d}) \\
\mathbf{E} & =\frac{1}{2}\left(\mathbf{F}^{\top} \mathbf{F}-\mathbf{I}\right) \\
\mathbf{S} & =\lambda \operatorname{trace}(\mathbf{E}) \mathbf{I}+2 \mu \mathbf{E} \\
\mathbf{\Pi} & =\mathbf{F S}
\end{aligned}
$$

where $\mathbf{S}$ is the second Piola-Kirchhoff stress tensor, $\mathbf{F}$ is the deformation gradient tensor, $J=\operatorname{det}(\mathbf{F})$ is the deformation gradients determinant, $\mathbf{E}$ is the Green-Lagrange strain tensor, $\Pi$ is the non-symmetric first Piola-Kirchhoff stress tensor and $\lambda$ and $\mu$ are the material Lame's constants. Then the equation of motion with respect to the initial configuration is given by

$$
\rho_{0} \frac{\partial^{2} \mathbf{d}}{\partial \mathrm{t}^{2}}=\nabla \cdot \boldsymbol{\Pi}+\rho_{0} \mathbf{b}
$$

where $\rho_{0}$ is the solid material density per unit undeformed volume.

\subsection{Interface conditions}

A main requirement for the coupling schemes is to fulfill two coupling conditions: the kinematic and the dynamic continuity across the fluid-solid interface at all times. The kinematic boundary conditions on the fluid-structue interface is driven by requiring continuity of the velocity

$$
\mathbf{u}=\dot{\mathbf{d}}
$$

while the dynamic condition means that the following equilibrium equation holds for the surface traction at the common fluid-structure interaction boundary

$$
\sigma_{s} \mathbf{n}_{s}=-\sigma_{f} \mathbf{n}_{f}
$$

where $\mathbf{n}_{s}$ and $\mathbf{n}_{f}$ denote the outward-pointing unit normal on the fluid-structure boundary, viewed from the structure and fluid domains, respectively. Here, $\sigma_{s}$ represents the Cauchy stress tensor of the structural field and $\sigma_{f}$ is the stress tensors in the case of an incompressible Newtonian fluid. The constitutive relation for the fluid stress tensor is given by

$$
\sigma_{f}=-p \mathbf{I}+\mu\left(\nabla \mathbf{u}+\nabla \mathbf{u}^{\top}\right)
$$

where $\mu$ is the fluid dynamic viscosity.

\subsection{Numerical discretization of ALE formulation for fluid domain}

The momentum equations along the $x-, y-$ and $z$-directions are discretized over the dual finite volume shown in Figure 1 and the dual volume involves only the right and left elements that share the common face where the components of the velocity vector are discretized. The discrete contribution from the right cell shown in Figure 1 is given below for each term of the momentum equation along the $x$-direction. The time derivative

$R e\left[\frac{3 u_{1}^{n+1}}{4 \Delta t}+\frac{\sum_{i} u_{i}^{n+1}}{6 \cdot 4 \Delta t}\right] V_{12345}-R e\left[\frac{3 u_{1}^{n}}{4 \Delta t}+\frac{\sum_{i} u_{i}^{n}}{6 \cdot 4 \Delta t}\right] V_{12345} \quad$ with $\quad i=1,2,3,4,5,10(13)$ 
The convective term due to fluid motion

$$
\begin{aligned}
& \frac{1}{2} \operatorname{Re}\left[\mathbf{u}_{125}^{n} \cdot \mathbf{A}_{125}^{n+1}\right] u_{125}^{n+1}+\frac{1}{2} \operatorname{Re}\left[\mathbf{u}_{125}^{n} \cdot \mathbf{A}_{125}^{n}\right] u_{125}^{n+1} \\
+ & \frac{1}{2} \operatorname{Re}\left[\mathbf{u}_{235}^{n} \cdot \mathbf{A}_{235}^{n+1}\right] u_{235}^{n+1}+\frac{1}{2} \operatorname{Re}\left[\mathbf{u}_{235}^{n} \cdot \mathbf{A}_{235}^{n}\right] u_{235}^{n+1} \\
+ & \frac{1}{2} \operatorname{Re}\left[\mathbf{u}_{345}^{n} \cdot \mathbf{A}_{345}^{n+1}\right] u_{345}^{n+1}+\frac{1}{2} \operatorname{Re}\left[\mathbf{u}_{345}^{n} \cdot \mathbf{A}_{345}^{n}\right] u_{345}^{n+1} \\
+ & \frac{1}{2} \operatorname{Re}\left[\mathbf{u}_{415}^{n} \cdot \mathbf{A}_{415}^{n+1}\right] u_{415}^{n+1}+\frac{1}{2} \operatorname{Re}\left[\mathbf{u}_{415}^{n} \cdot \mathbf{A}_{415}^{n}\right] u_{415}^{n+1}
\end{aligned}
$$

The convective term due to mesh motion

$$
\begin{aligned}
& -\frac{1}{2} \operatorname{Re}\left[\dot{\mathbf{x}}_{125}^{n+1} \cdot \mathbf{A}_{125}^{n+1}\right] u_{125}^{n}-\frac{1}{2} \operatorname{Re}\left[\dot{\mathbf{x}}_{125}^{n+1} \cdot \mathbf{A}_{125}^{n}\right] u_{125}^{n} \\
& -\frac{1}{2} \operatorname{Re}\left[\dot{\mathbf{x}}_{235}^{n+1} \cdot \mathbf{A}_{235}^{n+1}\right] u_{235}^{n}-\frac{1}{2} \operatorname{Re}\left[\dot{\mathbf{x}}_{235}^{n+1} \cdot \mathbf{A}_{235}^{n}\right] u_{235}^{n} \\
& -\frac{1}{2} \operatorname{Re}\left[\dot{\mathbf{x}}_{345}^{n+1} \cdot \mathbf{A}_{345}^{n+1}\right] u_{345}^{n}-\frac{1}{2} \operatorname{Re}\left[\dot{\mathbf{x}}_{345}^{n+1} \cdot \mathbf{A}_{345}^{n}\right] u_{345}^{n} \\
& -\frac{1}{2} \operatorname{Re}\left[\dot{\mathbf{x}}_{415}^{n+1} \cdot \mathbf{A}_{415}^{n+1}\right] u_{415}^{n}-\frac{1}{2} \operatorname{Re}\left[\dot{\mathbf{x}}_{415}^{n+1} \cdot \mathbf{A}_{415}^{n}\right] u_{415}^{n}
\end{aligned}
$$

The pressure term

$$
\begin{aligned}
& {\left[\frac{p_{1}+p_{2}+p_{5}}{3}\right]^{n+1} \mathbf{A}_{125}^{n+1} \cdot \mathbf{i}+\left[\frac{p_{2}+p_{3}+p_{5}}{3}\right]^{n+1} \mathbf{A}_{235}^{n+1} \cdot \mathbf{i} } \\
+ & {\left[\frac{p_{3}+p_{4}+p_{5}}{3}\right]^{n+1} \mathbf{A}_{345}^{n+1} \cdot \mathbf{i}+\left[\frac{p_{4}+p_{1}+p_{5}}{3}\right]^{n+1} \mathbf{A}_{415}^{n+1} \cdot \mathbf{i} }
\end{aligned}
$$

The viscous term

$$
\begin{aligned}
& -\left[\left(\frac{\partial u}{\partial x}\right)_{125}^{n+1} \mathbf{A}_{125}^{n+1} \cdot \mathbf{i}+\left(\frac{\partial u}{\partial y}\right)_{125}^{n+1} \mathbf{A}_{125}^{n+1} \cdot \mathbf{j}+\left(\frac{\partial u}{\partial z}\right)_{125}^{n+1} \mathbf{A}_{125}^{n+1} \cdot \mathbf{k}\right] \\
& -\left[\left(\frac{\partial u}{\partial x}\right)_{235}^{n+1} \mathbf{A}_{235}^{n+1} \cdot \mathbf{i}+\left(\frac{\partial u}{\partial y}\right)_{235}^{n+1} \mathbf{A}_{235}^{n+1} \cdot \mathbf{j}+\left(\frac{\partial u}{\partial z}\right)_{235}^{n+1} \mathbf{A}_{235}^{n+1} \cdot \mathbf{k}\right] \\
& -\left[\left(\frac{\partial u}{\partial x}\right)_{345}^{n+1} \mathbf{A}_{345}^{n+1} \cdot \mathbf{i}+\left(\frac{\partial u}{\partial y}\right)_{345}^{n+1} \mathbf{A}_{345}^{n+1} \cdot \mathbf{j}+\left(\frac{\partial u}{\partial z}\right)_{345}^{n+1} \mathbf{A}_{345}^{n+1} \cdot \mathbf{k}\right] \\
& -\left[\left(\frac{\partial u}{\partial x}\right)_{415}^{n+1} \mathbf{A}_{415}^{n+1} \cdot \mathbf{i}+\left(\frac{\partial u}{\partial y}\right)_{415}^{n+1} \mathbf{A}_{415}^{n+1} \cdot \mathbf{j}+\left(\frac{\partial u}{\partial z}\right)_{415}^{n+1} \mathbf{A}_{415}^{n+1} \cdot \mathbf{k}\right]
\end{aligned}
$$

where $V_{12345}$ is the volume of the pyramid between the points $\mathbf{x}_{1}, \mathbf{x}_{2}, \mathbf{x}_{3}, \mathbf{x}_{4}$ and $\mathbf{x}_{5}$ shown in Figure 1, $\mathbf{A}_{125}, \mathbf{A}_{235}, \mathbf{A}_{345}$ and $\mathbf{A}_{415}$ are the area vectors of the dual volume triangular surfaces, $\Delta t$ is the time step, the values $\mathbf{u}_{125}, \mathbf{u}_{235}, \mathbf{u}_{345}$ and $\mathbf{u}_{415}$ are the velocity vectors defined at the mid-point of each dual volume area and $p_{1}, p_{2}, p_{3}, p_{4}$ and $p_{5}$ are the pressure values at the points $\mathbf{x}_{1}, \mathbf{x}_{2}, \mathbf{x}_{3}, \mathbf{x}_{4}$ and $\mathbf{x}_{5}$, respectively. However, the pressure values at $\mathbf{x}_{1}$, 
$\mathbf{x}_{2}, \mathbf{x}_{3}$ and $\mathbf{x}_{4}$ are not known. To compute the pressure at $\mathbf{x}_{1}$, as an example, a second-order Taylor series expansion can be written as

$$
p_{i}=p_{1}+\left.\frac{\partial p}{\partial x}\right|_{\mathbf{x}=\mathbf{x}_{1}}\left(x_{i}-x_{1}\right)+\left.\frac{\partial p}{\partial y}\right|_{\mathbf{x}=\mathbf{x}_{1}}\left(y_{i}-y_{1}\right)+\left.\frac{\partial p}{\partial z}\right|_{\mathbf{x}=\mathbf{x}_{1}}\left(z_{i}-z_{1}\right)
$$

where $i$ represents the neighboring hexahedral elements connected to the $\mathrm{x}_{1}$ point. This overdetermined system of linear equations may be solved in a least square sense using the normal equation approach, in which both sides are multiplied by the transpose. The modified system is solved using the singular value decomposition provided by the Intel Math Kernel Library in order to avoid the numerical difficulties associated with solving linear systems with near rank deficiency. The velocity components are also computed at the points $\mathbf{x}_{1}, \mathbf{x}_{2}, \mathbf{x}_{3}$ and $\mathbf{x}_{4}$ in a similar manner. The velocity vector gradient components defined at the mid-point of each dual volume faces are computed by the use of the Green-Gauss theorem:

$$
\nabla u=\frac{\partial u}{\partial x} \mathbf{i}+\frac{\partial u}{\partial y} \mathbf{j}+\frac{\partial u}{\partial z} \mathbf{k}=\frac{1}{V_{C}} \oint_{\partial \Omega_{c}} u d \mathbf{A}
$$

where $V_{C}$ covolume consists two tetrahedral elements that share the same dual volume surface area and have their fourth vertices at the upwind and downwind velocity locations of the same dual volume surface area (for $\mathbf{A}_{125}$, the locations where $\mathbf{u}_{1}$ and $\mathbf{u}_{2}$ are defined). The right-hand side of the equations (19) is evaluated using the mid-point rule on each of the covolume faces. It should be noted that for the present finite-volume surface integrals involve only triangular planar surfaces for the momentum equations. The convective velocity vector components $u_{125}, u_{235}, u_{345}$ and $u_{415}$ are computed using the least square interpolations [1, 4]. As an example,

$$
u_{125}=\beta\left[u_{1}+\nabla u_{1} \mathbf{r}_{1}\right]+(1-\beta)\left[u_{2}+\nabla u_{2} \mathbf{r}_{2}\right]
$$

where $\beta$ is a weight factor determining the type of convection scheme used, $\nabla u_{1}$ and $\nabla u_{2}$ are the gradients of velocity components where the $u_{1}$ and $u_{2}$ velocity components are defined and $\mathbf{r}_{1}$ and $\mathbf{r}_{2}$ are the distance vectors from the mid-point of the dual volume surface to the locations where the gradients of velocity components are computed. For evaluating the gradient terms, $\nabla u_{1}$ and $\nabla u_{2}$, a least square procedure is used in which the velocity data is assumed to behave linearly. In order to satisfy the geometric conservation law (GCL) at the discrete level, the grid velocity components are computed through the use of the first-order backward differences.

$$
\dot{\mathbf{x}}^{n+1}=\frac{\mathbf{x}^{n+1}-\mathbf{x}^{n}}{\Delta t}
$$

This will ensure that the numerical scheme preserve a uniform flow solution exactly independent of the mesh motion. In comparison to the staggered methods, the use of the both velocity components significantly simplifies the discretization of the governing equations as well as the implementation of physical boundary conditions. The discretization of the momentum equation along the $y-$ and $z$-direction follows very closely the ideas presented here. The continuity equation (2) is integrated within each hexahedral elements and evaluated using the mid-point rule on each of the element faces

$$
-\sum_{i=1}^{6}\left[u^{n+1} A_{x}\right]_{i}+\left[v^{n+1} A_{y}\right]_{i}+\left[w^{n+1} A_{z}\right]_{i}=0
$$


where $\mathbf{A}=A_{x} \mathbf{i}+A_{y} \mathbf{j}+A_{z} \mathbf{k}$ is the hexahedral element surface area vector and $u, v$ and $w$ are the velocity vector components defined at the mid-point of each hexahedral element face. The discretization of above equations leads to a saddle point problem of the form:

$$
\left[\begin{array}{cccc}
A_{11} & 0 & 0 & A_{14} \\
0 & A_{22} & 0 & A_{24} \\
0 & 0 & A_{33} & A_{34} \\
A_{41} & A_{42} & A_{43} & 0
\end{array}\right]\left[\begin{array}{c}
u \\
v \\
w \\
p
\end{array}\right]=\left[\begin{array}{c}
b_{1} \\
b_{2} \\
b_{3} \\
0
\end{array}\right]
$$

where, $A_{11}, A_{22}$ and $A_{33}$ are the convection diffusion operators, $\left(A_{14}, A_{24}, A_{34}\right)^{\top}$ is the pressure gradient operator and $\left(A_{41}, A_{42}, A_{43}\right)$ is the divergence operator. It should also be noted that on an uniform Cartesian mesh the multiplication of the matrices $B_{41} B_{14}+B_{42} B_{24}+$ $B_{43} B_{34}$ gives the classical five-point Laplace operator as in the MAC scheme [20] which is extremely important for the efficient implementation of the present preconditioned iterative solvers.

\subsection{Galerkin finite element discretization for solid domain}

The weak form of the equations can be obtained by multiplying the equation (9) by the test functions, and integrating over the volume of the element as follows

$$
\int_{\Omega_{s}} N_{i} \rho \frac{\partial^{2} \mathbf{d}}{\partial \mathrm{t}^{2}} d V_{0}=\int_{\Omega_{s}} N_{i}(\nabla \cdot \boldsymbol{\Pi}+\rho \mathbf{b}) d V_{0}
$$

Integrating by parts one has

$$
\int_{\Omega_{s}} N_{i} \rho \frac{\partial^{2} \mathbf{d}}{\partial \mathrm{t}^{2}} d V_{0}=\int_{\Omega_{s}} \nabla \cdot\left(N_{i} \boldsymbol{\Pi}\right) d V_{0}-\int_{\Omega_{s}} \Pi \nabla N_{i} d V_{0}+\int_{\Omega_{s}} N_{i} \rho \mathbf{b} d V_{0}
$$

The first term can be related to a surface integral by the divergence theorem over the closed surface of the element, and the weak form in tensor notation can be obtained as

$$
\int_{\Omega_{s}} N_{i} \rho \frac{\partial^{2} \mathbf{d}}{\partial \mathrm{t}^{2}} d V_{0}=\oint_{\partial \Omega_{s}}\left(N_{i} \boldsymbol{\Pi}\right) \mathbf{n} d S_{0}-\int_{\Omega_{s}} \Pi \nabla N_{i} d V_{0}+\int_{\Omega_{s}} N_{i} \rho \mathbf{b} d V_{0}
$$

The displacements at any point in the isoparametric hexahedral element are approximated by a linear combination of the displacements at the nodal points of the element

$$
\left\{\begin{array}{l}
d_{x} \\
d_{y} \\
d_{z}
\end{array}\right\}=\left[\begin{array}{ccccccccccccc}
N_{1} & 0 & 0 & N_{2} & 0 & 0 & N_{3} & 0 & 0 & \cdots & N_{8} & 0 & 0 \\
0 & N_{1} & 0 & 0 & N_{2} & 0 & 0 & N_{3} & 0 & \cdots & 0 & N_{8} & 0 \\
0 & 0 & N_{1} & 0 & 0 & N_{2} & 0 & 0 & N_{3} & \cdots & 0 & 0 & N_{8}
\end{array}\right]\left\{\begin{array}{c}
d_{x 1} \\
d_{y 1} \\
d_{z 1} \\
\vdots \\
d_{x 8} \\
d_{y 8} \\
d_{z 8}
\end{array}\right\}=\mathbf{N d}
$$

The stiffness matrix for the hexahedral element is obtained from the discretization of the third integral term of the weak form of the equations (26).

$$
\int_{\Omega_{s}} \Pi \nabla N_{i} d V_{0}=\int_{\Omega_{s}} \boldsymbol{F} \boldsymbol{S} \nabla N_{i} d V_{0}=\int_{\Omega_{s}}(\mathbf{I}+\nabla \mathbf{d}) \boldsymbol{S} \nabla N_{i} d V_{0}
$$


The operation of the second Piola-Kirchhoff stress tensor on $\nabla N_{i}$ can explicitly given in matrix notation as

$$
\boldsymbol{S} \nabla N_{i}=\left[B_{i}\right]^{\top}\{S\}
$$

The integral can be obtained, at this stage, as

$$
\int_{\Omega_{s}} \Pi \nabla N_{i} d V_{0}=\int_{\Omega_{s}}(\mathbf{I}+\nabla \mathbf{d})\left[B_{i}\right]^{\top}\{S\} d V_{0}
$$

where

$$
(\mathbf{I}+\nabla \mathbf{d})\left[B_{i}\right]^{\top}=\left[B_{i}\right]^{\top}+\nabla \mathbf{d}\left[B_{i}\right]^{\top}=\left[B_{i}\right]^{\top}+\left[B_{i}\right]_{N L}^{\top}
$$

The second Piola-Kirchhoff stress tensor can be written as

$$
\left\{\begin{array}{c}
S_{x x} \\
S_{y y} \\
S_{z z} \\
S_{x y} \\
S_{x z} \\
S_{y z}
\end{array}\right\}=\underbrace{\left[\begin{array}{cccccc}
\lambda+2 \mu & \lambda & \lambda & 0 & 0 & 0 \\
\lambda & \lambda+2 \mu & \lambda & 0 & 0 & 0 \\
\lambda & \lambda & \lambda+2 \mu & 0 & 0 & 0 \\
0 & 0 & 0 & \mu & 0 & 0 \\
0 & 0 & 0 & 0 & \mu & 0 \\
0 & 0 & 0 & 0 & 0 & \mu
\end{array}\right]}_{\mathbf{C}}\left\{\begin{array}{c}
E_{x x} \\
E_{y y} \\
E_{z z} \\
2 E_{x y} \\
2 E_{x z} \\
2 E_{y z}
\end{array}\right\}
$$

where the Green-St. Venant strain tensor can be obtained in vector form using the definition given in (5) and (6). Defining the displacement field in terms of the nodal displacements using (27), the the Green-St. Venant strain tensor in vector form can be obtained as

$$
\{E\}=\left[\mathbf{B}+\frac{1}{2} \mathbf{B}_{N L}\right]\{\mathbf{d}\}
$$

Hence, the element stiffness matrix can be calculated as

$$
\mathbf{K}_{(e)}=\int_{\Omega_{s}}\left(\mathbf{B}+\mathbf{B}_{N L}\right)^{\top} \mathbf{C}\left(\mathbf{B}+\frac{1}{2} \mathbf{B}_{N L}\right) d V_{0}
$$

It should be noted that we do not use the Newton's method here. The element mass matrix can be obtained from the inertial term in the equation (26) by substituting the displacements equation (27) one has

$$
\mathbf{M}_{(e)}=\int_{\Omega_{s}} \rho \mathbf{N}^{T} \mathbf{N} d V_{0}
$$

Finally, the boundary loads are imposed on the face of the surface elements by the surface integral term given in the equation (26)

$$
\oint_{\partial \Omega_{s}} N_{i}\left[\begin{array}{ccc}
\Pi_{x x} & \Pi_{x y} & \Pi_{x z} \\
\Pi_{y x} & \Pi_{y y} & \Pi_{y z} \\
\Pi_{z x} & \Pi_{z y} & \Pi_{z z}
\end{array}\right]\left\{\begin{array}{l}
n_{x} \\
n_{y} \\
n_{z}
\end{array}\right\} d S_{0}=\oint_{\partial \Omega_{s}} N_{i}\left[\begin{array}{ccc}
\sigma_{x x} & \sigma_{x y} & \sigma_{x z} \\
\sigma_{y x} & \sigma_{y y} & \sigma_{y z} \\
\sigma_{z x} & \sigma_{z y} & \sigma_{z z}
\end{array}\right]_{s}\left\{\begin{array}{l}
\hat{n}_{x} \\
\hat{n}_{y} \\
\hat{n}_{z}
\end{array}\right\} d S
$$

where $\hat{\mathbf{n}}$ is the surface normal vector given in the deformed solid domain

$$
\hat{\mathbf{n}}=\hat{n}_{x} \mathbf{i}+\hat{n}_{y} \mathbf{j}+\hat{n}_{z} \mathbf{k}
$$

Then the following equilibrium equation is applied at the common fluid-structure interaction boundary

$$
\sigma_{s} \hat{\mathbf{n}}_{s}=-\sigma_{f} \hat{\mathbf{n}}_{f}
$$


Upon assembly of the element matrices the following dynamic system of equations can be obtained for the structure.

$$
\mathbf{M} \ddot{\mathbf{d}}+\mathbf{R}(\mathbf{d})=\mathbf{F}
$$

where $\mathbf{M}, \mathbf{R}(\mathbf{d})$ and $\mathbf{F}$ are the global mass matrix, nonlinear residual due to material stiffness and load vector respectively, and $\mathbf{d}$ is the vector of global displacements. In the present approach the system given in (39) is solved using the generalized- $\alpha$ method of Chung and Hulbert [9]. The generalized $-\alpha$ method is an implicit, onestep time integration scheme based on Newmark like approximations [28] in the time domain.

\subsection{Mesh deformation algorithm}

A FSI algorithm requires a scheme for moving mesh vertices as the fluid-structure interfaces deform, rotate and translate. Several mesh deforming algorithms have been presented in the literature including the spring analogy [5], the elastic medium analogy [25], the edge swapping algorithm [12] and the remeshing algorithm [26]. These methods generally require solving discrete equations using iterative methods and the computational effort for these methods in three-dimension is not negligible. Therefore, we employ an alternative algebraic method [18] which assumes an exponential decay of the mesh displacement away from the fluid-structure interaction based on the minimum distance function. In addition, the equations of linear elasticity can be solved for more difficult mesh deformations and the robustness of the method can be further improved by modifying the Youngs module based on the distance function in order to increase the stif! fness of small elements close to the fluid-structure interaction [34].

\subsection{Coupled System of Equations}

When the fluid and structure solvers are coupled with the interface conditions given in the section 2.3 and the mesh deformation algorithm described in the section 2.6, the following linear algebraic system of equations are reached.

$$
\left[\begin{array}{cc|c|cc|c}
A_{u u} & A_{u u_{\Gamma}} & A_{u p} & 0 & 0 & A_{u q} \\
0 & A_{u_{\Gamma} u_{\Gamma}} & 0 & 0 & A_{u d_{\Gamma}} & 0 \\
\hline A_{p u} & A_{p u_{\Gamma}} & 0 & 0 & 0 & 0 \\
\hline 0 & 0 & 0 & A_{d d} & A_{d d_{\Gamma}} & 0 \\
A_{d_{\Gamma} u} & A_{d_{\Gamma} u_{\Gamma}} & A_{d_{\Gamma} p} & A_{d_{\Gamma} d} & A_{d_{\Gamma} d_{\Gamma}} & 0 \\
\hline 0 & 0 & 0 & 0 & A_{q d_{\Gamma}} & A_{q q}
\end{array}\right]\left[\begin{array}{c}
\mathbf{u} \\
\mathbf{u}_{\Gamma} \\
\hline p \\
\hline \mathbf{d} \\
\mathbf{d}_{\Gamma} \\
\hline \mathbf{q}
\end{array}\right]=\left[\begin{array}{c}
b_{1} \\
0 \\
\hline 0 \\
\hline b_{4} \\
b_{5} \\
\hline 0
\end{array}\right]
$$

where $\Gamma$ represents the variables at the common fluid-structure interface, $q$ is the amount of mesh deformation within the fluid domain. These equations can be written as:

$$
\left[\begin{array}{cccc}
A_{u u} & A_{u p} & A_{u d} & A_{u q} \\
A_{p u} & 0 & 0 & 0 \\
A_{d u} & A_{d p} & A_{d d} & 0 \\
0 & 0 & A_{q d} & A_{q q}
\end{array}\right]\left[\begin{array}{c}
\mathbf{u} \\
p \\
\mathbf{d} \\
\mathbf{q}
\end{array}\right]=\left[\begin{array}{c}
d_{1} \\
0 \\
d_{3} \\
0
\end{array}\right]
$$

In practice, the solution of equation (41) does not converge very quickly and it is rather difficult to construct robust preconditioners for the whole coupled system because of the zeroblock diagonal resulting from the divergence-free constraint. In the present paper, we use an 
upper triangular right preconditioner which results in a scaled discrete Laplacian instead of a zero block in the original system. Then the modified system becomes

$$
\left[\begin{array}{cccc}
A_{u u} & A_{u p} & A_{u d} & A_{u q} \\
A_{p u} & 0 & 0 & 0 \\
A_{d u} & A_{d p} & A_{d d} & 0 \\
0 & 0 & A_{q d} & A_{q q}
\end{array}\right]\left[\begin{array}{cccc}
I & \hat{A}_{u p} & 0 & 0 \\
0 & I & 0 & 0 \\
0 & 0 & I & 0 \\
0 & 0 & 0 & I
\end{array}\right]=\left[\begin{array}{cccc}
A_{u u} & A_{u u} \hat{A}_{u p}+A_{u p} & A_{u d} & A_{u q} \\
A_{p u} & A_{p u} \hat{A}_{u p} & 0 & 0 \\
A_{d u} & A_{d u} \hat{A}_{u p}+A_{d p} & A_{d d} & 0 \\
0 & 0 & A_{q d} & A_{q q}
\end{array}\right]
$$

and the zero block is replaced with $-A_{p u} A_{u p}$, which is a scaled discrete Laplacian. Unfortunately, this leads to a significant increase in the number of non-zero elements due to the matrix-matrix multiplication. However, it is possible to replace the $-A_{u p}$ block matrix in the upper triangular right preconditioner with a computationally less expensive matrix, $-\hat{A}_{u p}$. The calculations indicate that the largest contribution for the pressure gradients in the momentum equations comes from the right and left elements that share the common edge/face where the components of the velocity vector are discretized. Therefore, we will use the contribution from these two elements for the $-\hat{A}_{u p}$ matrix which leads maximum three non-zero entries per row. Although, this approximation does not change the convergence rate of an iterative solver significantly, it leads to a significant reduction in the computing time and memory requirement. The present one-level iter! ative solver is based on the restricted additive Schwarz method with the flexible GMRES $(m)$ algorithm. Since the zero block is removed, a blockincomplete factorization coupled with the reverse Cuthill-McKee ordering [11] can be used within each partitioned sub-domains.

Multigrid methods $[19,36]$ are known to be the most efficient numerical techniques for solving large-scale problems that arise in numerical simulations of physical phenomena because of their computational costs and memory requirements that scale linearly with the degrees of freedom. The two level iterative iterative solver based on the multiplicative nonnested multigrid method with one V-cycle is described in [33] in detail and it has been successfully applied to the solution of the Stokes problem. However, this solver is not suitable for time-dependent calculations with small time steps since the resulting mass matrices are highly diagonal. Therefore, a matrix factorization is introduced similar to that of the projection method [10]

$$
\left[\begin{array}{cccc}
I & A_{u p} & 0 & 0 \\
A_{p u} & 0 & 0 & 0 \\
0 & 0 & I & 0 \\
0 & 0 & A_{q d} & I
\end{array}\right]=\left[\begin{array}{cccc}
I & 0 & 0 & 0 \\
A_{p u} & I & 0 & 0 \\
0 & 0 & I & 0 \\
0 & 0 & A_{q d} & I
\end{array}\right]\left[\begin{array}{cccc}
I & 0 & 0 & 0 \\
0 & -A_{p u} A_{u p} & 0 & 0 \\
0 & 0 & I & 0 \\
0 & 0 & 0 & I
\end{array}\right]\left[\begin{array}{cccc}
I & A_{u p} & 0 & 0 \\
0 & I & 0 & 0 \\
0 & 0 & I & 0 \\
0 & 0 & 0 & I
\end{array}\right]
$$

Then the preconditioner matrix becomes

$$
\left[\begin{array}{cccc}
I & -A_{u p} & 0 & 0 \\
0 & I & 0 & 0 \\
0 & 0 & 1 & 0 \\
0 & 0 & 0 & 1
\end{array}\right]\left[\begin{array}{cccc}
I & 0 & 0 & 0 \\
0 & \left(-A_{p u} A_{u p}\right)^{-1} & 0 & 0 \\
0 & 0 & I & 0 \\
0 & 0 & 0 & I
\end{array}\right]\left[\begin{array}{cccc}
I & 0 & 0 & 0 \\
-A_{p u} & I & 0 & 0 \\
0 & 0 & I & 0 \\
0 & 0 & -A_{q d} & I
\end{array}\right]
$$

For the inverse of the scaled discrete Laplacian, we use two-cycle of BoomerAMG solver provided by the HYPRE library [17], a high performance preconditioning package developed at Lawrence Livermore National Laboratory, which we access through the PETSc library. Although we solve the whole coupled system with the above preconditioner, the matrices are stored at the block level. The implementation of the preconditioned Krylov subspace algorithm and matrix-matrix multiplication were carried out using the PETSc [2] software package 
developed at the Argone National Laboratories. METIS library [27] is used to decompose the flow domain into a set of sub-domains.

\section{NUMERICAL EXPERIMENTS}

In this section, the proposed FSI algorithm described in Section 2 is initially validated for an unsteady Newtonian fluid interacting with an elastic rectangular bar behind a circular cylinder and a three-dimensional elastic solid confined in a rectangular channel.

\subsection{Test Case I: Fluid-structure interaction of an elastic bar behind a rigid cylinder}

The first case corresponds to the FSI benchmark problem proposed by Hron and Turek [22]. The problem consists of an elastic bar behind a rigid circular cylinder which is placed asymmetrically between parallel lateral walls as shown in Figure 2.

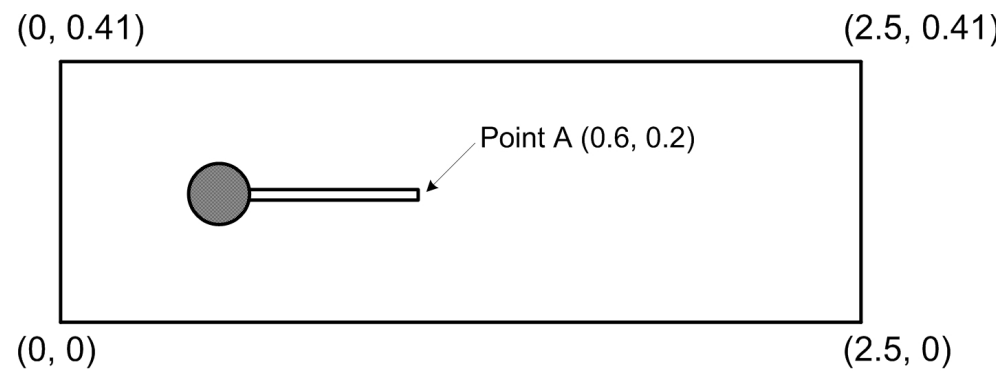

Figure 2. The geometric description of the first validation case.

The parameters $H=0.41$ is the $2 \mathrm{D}$ channel height, $L=2.5$ is the channel length, $D=0.1$ is the cylinder diameter, $l=0.35$ is the bar length and $h=0.02$ is the bar height. The cylinder is positioned at $(0.2,0.2)$ from the left bottom corner of the channel. The control point $A$ is attached to the structure and moving in time starting from $(0.6,0.2)$. The inflow velocity is set to

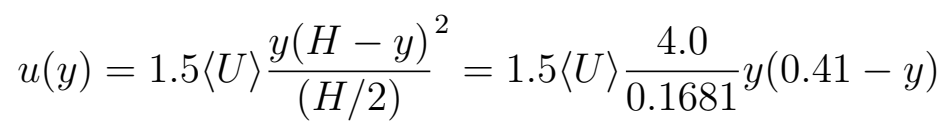

where $\langle U\rangle$ is the mean inflow velocity. The outlet boundary conditions are set to natural (traction-free) boundary conditions:

$$
\frac{\partial u}{\partial x}=p, \quad \frac{\partial v}{\partial x}=0
$$

We consider two cases with two different inflow speeds and shear moduli for the solid for this test problem with the physical parameters indicated in Table 1. FSI1, where the Reynolds number $R e=20$, results in a steady state solution, while FSI2, which corresponds to FSI3 of Hron and Turek [22], results in an unsteady flow solution with the Reynolds number $R e=200$.

For the present test case, we use an unstructured mesh with 78,921 quadrilateral elements and 79,806 nodes, which result in a total of 375,216 DOF for the whole domain. The mesh is highly refined close to the solid surfaces using local mesh refinement algorithms within the CUBIT [7] library. The computed $u$-velocity vector components for FSI2 are 
Table 1. Fluid and structure properties for Test Case I

\begin{tabular}{llll}
\hline & & FSI1 & FSI2 \\
\hline \hline \multirow{3}{*}{ Fluid } & Density $\rho_{f}$ & 1000 & 1000 \\
\cline { 2 - 4 } & Dynamic viscosity $\mu_{f}$ & $1 \times 10^{-3}$ & $1 \times 10^{-3}$ \\
\cline { 2 - 4 } Solid & Mean Inflow velocity $\langle U\rangle$ & 0.2 & 2 \\
\hline \multirow{2}{*}{} & Density $\rho_{s}$ & 1000 & 1000 \\
\cline { 2 - 4 } & Poisson ratio $\nu_{s}$ & 0.4 & 0.4 \\
\cline { 2 - 4 } & Shear modulus $\mu_{s}$ & $0.5 \times 10^{6}$ & $2 \times 10^{6}$ \\
\hline
\end{tabular}

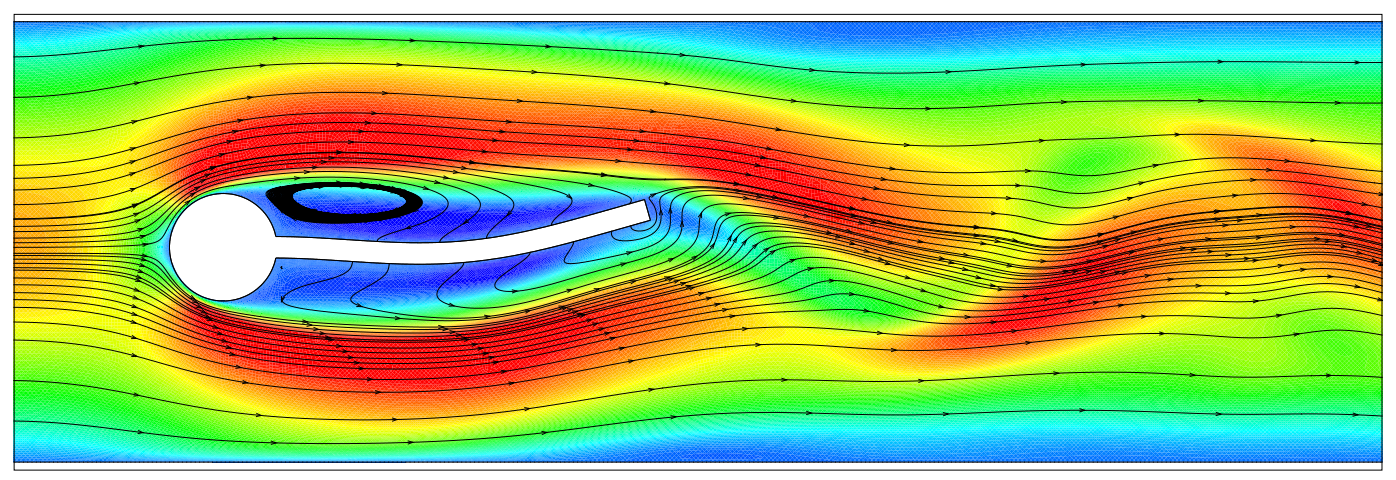

Figure 3. The computed $u$-velocity vector component contours with the streamlines for an elastic bar behind a rigid cylinder at $R e=200$.

shown in Figure 3 with the streamlines at $t=10.09$. The streamlines indicate an alternating large recirculation zone just behind the cylinder.

The time variation of vertical displacement for FSI2 at point $A(0.6,0.2)$ on the tip of the bar is given in Figure 4. The computed deformations at point $A$ for the steady and the unsteady flow solutions are tabulated in Table 2 and 3 with comparisons to the computations from different authors. The present results are in relative good agreement with the results in the literature and the present calculations can correctly predict the amplitude of the oscillations for the unsteady flow solution (FSI2).

Table 2. Displacements at point $A$ for FSI1 of Test Case I. $R e=20$.

\begin{tabular}{llll}
\hline & DOF & $d_{x}\left[\times 10^{-3}\right]$ & $d_{y}\left[\times 10^{-3}\right]$ \\
\hline \hline Present FSI solver & 375,216 & 0.022387 & 0.81408 \\
\hline Hron and Turek [22] & 304,128 & 0.022732 & 0.82071 \\
\hline Degroote et al. [38] & 320,372 & 0.022651 & 0.83478 \\
\hline Richter and Wick [39] & 351,720 & 0.022695 & 0.81556 \\
\hline
\end{tabular}


Table 3. Displacements at point $A$ for FSI2 of Test Case I. $R e=200$.

\begin{tabular}{llll}
\hline & DOF & $d_{x}\left[\times 10^{-3}\right]$ & $d_{y}\left[\times 10^{-3}\right]$ \\
\hline \hline Present FSI solver & 375,216 & $-2.521 \pm 2.655$ & $+1.327 \pm 33.659$ \\
\hline Hron and Turek [22] & 304,128 & $-2.69 \pm 2.53$ & $+1.48 \pm 34.38$ \\
\hline Wick [40] & 72,696 & $-2.84 \pm 2.67$ & $+1.28 \pm 34.61$ \\
\hline Chabannes et al. [41] & 86,256 & $-2.90 \pm 2.77$ & $+1.33 \pm 34.90$ \\
\hline
\end{tabular}

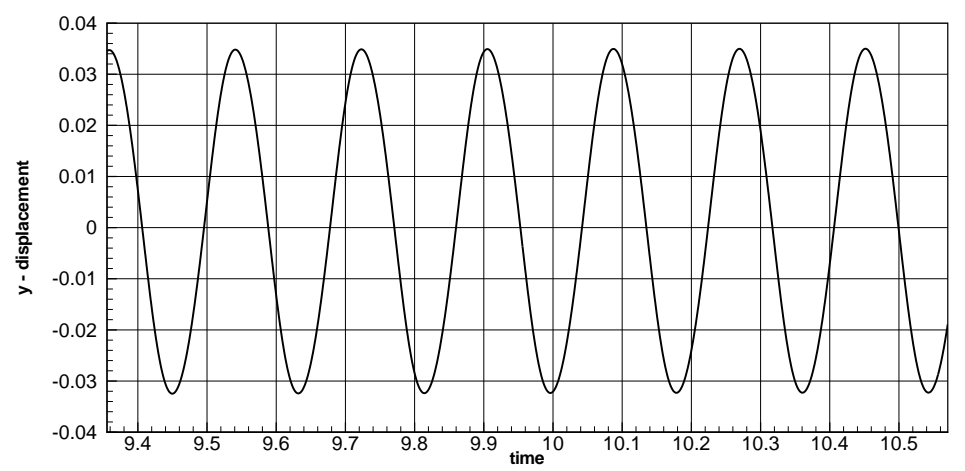

Figure 4. The time variation of vertical displacement for an elastic bar behind a rigid cylinder at $R e=200$.

\subsection{Test Case II: 3D FSI problem of an elastic beam in a cross flow}

In the second example, the present FSI solver is validated for a 3-dimensional stationary problem, where an elastic beam is immersed in a rectangular channel as illustrated in Figure 5. The dimensions of the channel is $[0,1.5] \times[0,0.4] \times[-0.4,0.4]$ while the dimensions of solid domain is $[0.4,0,5] \times[0,0.2] \times[-0.2,0.2]$. The problem is considered to be symmetric in the $x y$-plane. The present problem is solved by Richter [30] using a variational monolithic Arbitrary Lagrangian Eulerian formulation with local mesh refinement, resulting upto $7600775 \mathrm{DOF}$. The author provides the structure displacement values in $x-$ and $y$-direction at the point $A(0.45,0.15,0.15)$ and the drag forces on the solid body. The

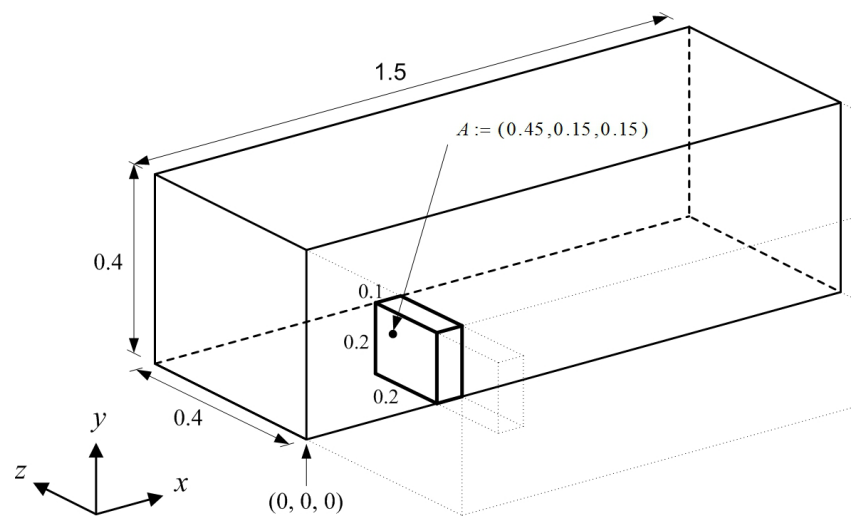

Figure 5. The geometry for 3D FSI problem. 
inlet velocity has a parabolic distribution given

$$
u(y, z)=\frac{0.3}{0.2^{2} \times 0.4^{2}} y(0.4-y)\left(0.4^{2}-z^{2}\right)
$$

with the maximum value of $u_{\max }=0.3$. The outlet boundary conditions are set to natural (traction-free) boundary conditions.

The Reynolds number is based on an average inflow velocity $(\langle U\rangle=0.2)$ and an obstacle of height $h=0.2$ and its value is $R e=40$. The properties for the fluid and the solid are tabulated in Table 4. The computational mesh consists of 362,224 hexahedral elements and 374,523 vertices leading to 4,096,6514 DOF. The mesh is initially constructed from a Cartesian mesh and then two levels of refinement are performed near the solid walls. The computed $u$-velocity vector component isosurfaces are shown in Figure 6 with the streamtraces at $R e=40$. The computed deformation vector components at the point $A(0.45,0.15,0.15)$ are given in Table 5 and the values are compared with the results of Richter [30]. The results are relatively in good agreement.

Table 4. Fluid and structure properties for test case II

\begin{tabular}{llll}
\hline Fluid & \multicolumn{3}{l}{ Structure } \\
\hline \hline Density & $\rho_{f}=1000$ & Density & $\rho_{s}=1000$ \\
\hline Kinematic viscosity & $\nu_{f}=10^{-3}$ & Poisson ratio & $\nu_{s}=0.4$ \\
\hline Average Inflow velocity & $\langle U\rangle=0.2$ & Shear Modulus & $\mu_{s}=5 \times 10^{5}$ \\
\hline
\end{tabular}

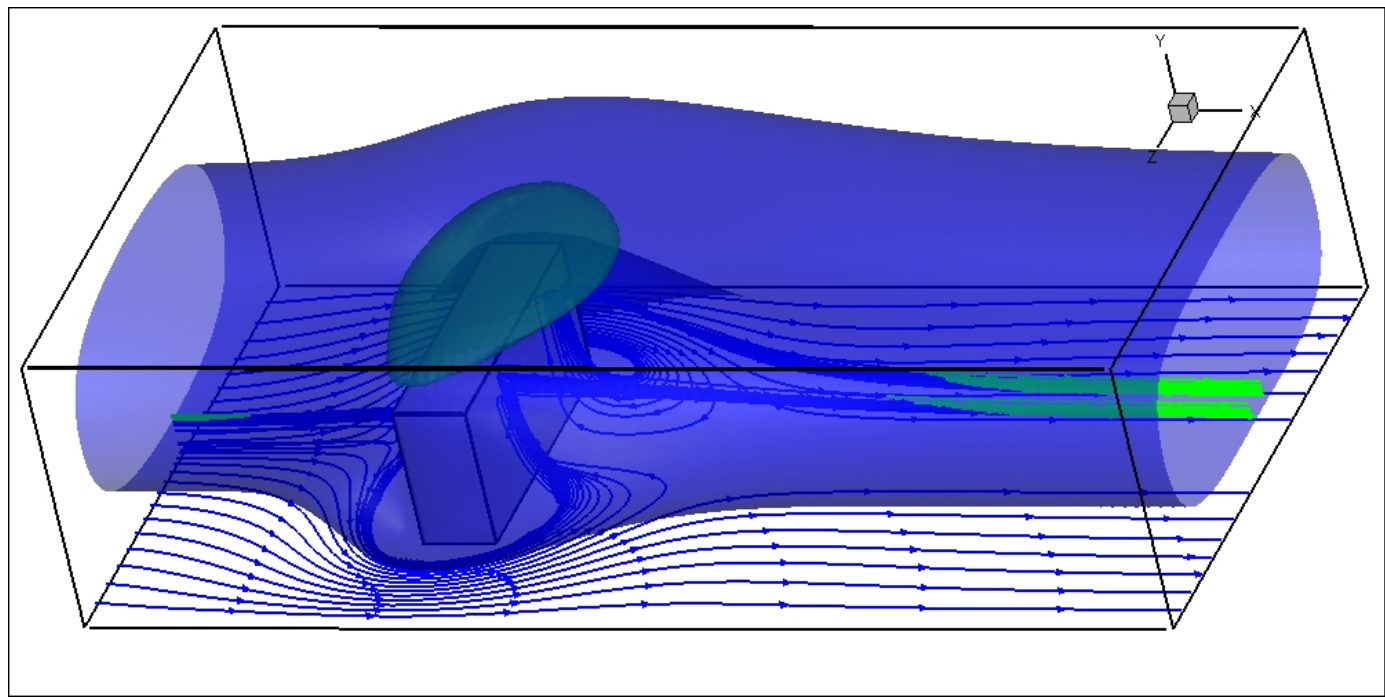

Figure 6. The computed $u$-velocity vector component isosurfaces with the streamtraces for an elastic bar confined in a rectangular channel at $R e=40$.

\section{CONCLUSIONS}

A new algorithm based on the ALE formulation is presented for a fully coupled solution of the large-scale FSI problems where the fluid is modeled by the incompressible NavierStokes equations and the structure is modeled by the St. Venant-Kirchhoff model. The present 
Table 5. Displacement at point $A$ for Test Case II

\begin{tabular}{lll}
\hline & Present FSI solver & Richter [30] \\
\hline \hline DOF & $4,096,651$ & $7,600,775$ \\
\hline$\Delta x$ & $5.754015 \times 10^{-5}$ & $5.9280 \times 10^{-5}$ \\
\hline$\Delta y$ & $8.749694 \times 10^{-7}$ & - \\
\hline$\Delta z$ & $8.532640 \times 10^{-7}$ & - \\
\hline
\end{tabular}

ALE formulation does not require any ad-hoc modifications in order to enhance the pressurevelocity coupling. The continuity equation is satisfied within each element exactly and the summation of the continuity equations can be exactly reduced to the domain boundary, which is important for the global mass conservation. A special attention is also given to satisfy the Geometric Conservation Law (GCL) on moving meshes at discrete level. The deformation of the solid domain is governed by the constitutive laws for the nonlinear Saint Venant-Kirchhoff material and the classical Galerkin finite element is used to discretize the governing equations in a Lagrangian frame. The implementation of the preconditione! $d$ coupled iterative solvers is based on the PETSc library for improving the efficiency of the parallel code. The present numerical algorithm is validated for a steady and unsteady Newtonian fluid interacting with an elastic rectangular bar behind a circular cylinder and a three-dimensional elastic solid confined in a rectangular channel. In the future, the numerical algorithm will be applied to investigate fluid-structure interaction of a membrane-wing micro aerial vehicle (MAV).

\section{Acknowledgements}

The authors gratefully acknowledge the use of the Chimera machine at the Faculty of Aeronautics and Astronautics at ITU, the computing resources provided by the National Center for High Performance Computing of Turkey (UYBHM) under grant number 10752009 and the computing facilities at TUBITAK ULAKBIM, High Performance and Grid Computing Center.

\section{References}

[1] W. K. Anderson and D. L. Bonhaus, An implicit upwind algorithm for computing turbulent flows on unstructured grids. Comp. \& Fluids 23, (1994), 1-21.

[2] S. Balay, K. Buschelman, V. Eijkhout, W. D. Gropp, D. Kaushik, M. G. Knepley, L. C. McInnes, B. F. Smith and H. Zhang, PETSc Users Manual. ANL-95/11, Mathematic and Computer Science Division, Argonne National Laboratory, (2004). http://wwwunix.mcs.anl.gov/petsc/petsc-2/

[3] A. T. Barker and X.-C. Cai, Scalable parallel methods for monolithic coupling in fluidstructure interaction with application to blood flow modeling. J. Comput.Phys. 229, (2010), 642-659.

[4] T. J. Barth, A 3-D upwind Euler solver for unstructured meshes. AIAA Paper 91-1548$C P,(1991)$.

[5] J. T. Batina. Unsteady Euler airfoil solutions using unstructured dynamic meshes. AIAA J. 28, (1990), 1381-1388. 
[6] M. Behr, T. E. Tezduyar, Finite element solution strategies for large-scale flow simulations. Comput. Methods Appl. Mech. Engrg. 112, (1994), 3-24.

[7] T. D. Blacker, S. Benzley, S. Jankovich, R. Kerr, J. Kraftcheck, R. Kerr, P. Knupp, R. Leland, D. Melander, R. Meyers, S. Mitchell, J. Shepard, T. Tautges and D. White, CUBIT Mesh Generation Enviroment Users Manual Volume 1. Sandia National Laboratories: Albuquerque, NM (1999).

[8] F. J. Blom, A monolithical fluid-structure interaction algorithm applied to the piston problem. Methods Appl. Mech. Engrg. 167, (1998), 369-391.

[9] J. Chung and G. M. Hulbert, A time integration algorithm for structural dynamics with improved numerical dissipation: the generalized- method. J. Appl. Math. 60, (1993), 371-375.

[10] A. J. Chorin, Numerical solution of the Navier-Stokes equations. Math. Comp. 22, (1968), 745-762.

[11] E. Cuthill and J. McKee, Reducing the bandwidth of sparce symmetric matrices. 24th. ACM National Conference, (1969), 157-172.

[12] M. Dai, D.P. Schmidt, Adaptive tetrahedral meshing in free-surface flow, J. Comput. Phy. 208 (2005), 228-252.

[13] J. Donea, S. Jiuliani, and J. P. Halleux. An arbitrary Lagrangian-Eulerian finite element method for transient dynamic fluid-structure interactions. Comp. Meth. Appl. Mech. Eng. 33, (1982), 689-723.

[14] C. Farhat. CFD-based nonlinear computational aeroelasticity. Encyclopedia of computational mechanics 3, (2004), 459-480, E. Stein, R. De Borst and T. Hughes (Eds), John Wiley \& Sons.

[15] M. W. Gee, U. Küttler and W. A. Wall, Truly monolithic algebraic multigrid for fluidstructure interaction. Int. J. Numer. Meth. Engng 85, (2011), 987-1016.

[16] M. Heil, A. L. Hazel and J. Boyle, Solvers for large-displacement fluidstructure interaction problems: segregated versus monolithic approaches. Comput. Mech. 43, (2008), 91-101.

[17] R. Falgout, A. Baker, E. Chow, V. E. Henson, E. Hill, J. Jones, T. Kolev, B. Lee, J. Painter, C. Tong, P. Vassilevski and U. M. Yang, Users manual, HYPRE High Performance Preconditioners. UCRL-MA-137155 DR, Center for Applied Scientific Computing, Lawrence Livermore National Laboratory, (2002). http://www.llnl.gov/CASC/hypre/

[18] T. Gerhold and J. Neumann, The parallel mesh deformation of the DLR TAU-code. New Results in Numerical and Experimental Fluid Mechanics VI 96, (2007), 162-169.

[19] W. Hackbusch, Multigrid methods and applications. Springer-Verlag, Heidelberg, (1985).

[20] F. H. Harlow and J. E. Welch, Numerical calculation of time-dependent viscous incompressible flow of fluid with free surface. J. Comput. Phys. 8, (1965), 2182-2189. 
[21] C. W. Hirt, A. A. Amsden and J. L. Cook, An arbitrary LagrangianEulerian computing method for all flow speeds. J. Comput. Phys. 14, (1974), 227-253.

[22] J. Hron, S. Turek, Proposal for numerical benchmarking of fluidstructure interaction between an elastic object and laminar incompressible flow. FluidStructure Interaction: Modeling, Simulation, Optimization, Lecture Notes in Computational Science and Engineering 53, (2006), Hans-Joachim Bungartz, Michael Schafer (Eds.), Springer, 146-170.

[23] T. J. R. Hughes, W. K. Liu, and T. Zimmerman, Lagrangian-Eulerian finite element formulation for incompressible viscous flow. Comp. Meth. Appl. Mech. Eng. 29,(1981), 329-349.

[24] Y. H. Hwang, Calculations of incompressible flow on a staggered triangle grid, Part I: Mathematical formulation. Numer. Heat Transfer B 27, (1995), 323-1995.

[25] A. A. Johnson, T. E. Tezduyar, Mesh update strategies in parallel finite element computations of flow problems with moving boundaries and interfaces. Comput. Methods Appl. Mech. Engrg. 119, (1994) 73-94.

[26] A. Johnson, T. Tezduyar, Advanced mesh generation and update methods for 3D flow simulations. Comput. Mech. 23, (1999) 130-143.

[27] G. Karypis and V. Kumar, A fast and high quality multilevel scheme for partitioning irregular graphs. SIAM J. Sci. Comput. 20, (1998), 359-392.

[28] N. M. Newmark, A method of computation for structural dynamics. Journal of the Engineering Mechanics Division (ASCE) 85, (1959), 67-94.

[29] K. C. Park, C. A. Felippa, and J. A. Deruntz. Stabilization of staggered solution procedures for fluidstructure interaction analysis. Computational Methods for Fluid-Structure Interaction Problems, (1977), 94-124, T. Belytschko and T. L. Geers, (Eds.), ASME.

[30] T. Richter, Goal-oriented error estimation for fluid-structure interaction problems. Comput. Methods Appl. Mech. Engrg., (submitted).

[31] S. Rida, F. McKenty, F. L. Meng and M. Reggio, A staggered control volume scheme for unstructured triangular grids. Int. J. Numer. Meth. Fluids 25, (1997), 697-717.

[32] Y. Saad, A flexible inner-product preconditioned GMRES algorithm. SIAM J. Sci. Statist. Comput. 14, (1993), 461-469.

[33] M. Sahin, A stable unstructured finite volume method for parallel large-scale viscoelastic fluid flow calculations. Journal of non-Newtonian Fluid Mechanics 166, (2011), 779_ 791.

[34] M. Sahin and K. Mohseni, An Arbitrary Lagrangian-Eulerian Formulation for the Numerical Simulation of Flow Patterns Generated by the Hydromedusa Aequorea Victoria. J. Comput. Phys. 228, (2009), 4588-4605.

[35] P. D. Thomas and C. K. Lombard, Geometric conservation law and its application to flow computations on moving grids. AIAA J. 17, (1979), 1030-1037. 
[36] P. Wesseling, An introduction to multigrid methods. John Wiley \& Sons, New York, (1992).

[37] E. L. Wilson, R. L. Taylor, W. P. Doherty and J. Ghaboussi, Incompatible displacement models. Numerical and Computer Methods in Structural Mechanics, S.J. Fenves et al., Eds., Academic Press, New York, (1973) 43-58.

[38] J. Degroote, R. Haelterman, S. Annerel, P. Bruggeman , J. Vierendeel, Performance of partitioned procedures in fluid-structure interaction. Comput Struct 88, (2010), 446-457.

[39] Th. Richter, Th. Wick, Finite elements for fuid-structure interaction in ALE and fully Eulerian coordinates. Comput. Methods Appl. Mech. Engrg. 199, (2010), 2633-2642.

[40] Th. Wick, Solving Monolithic Fluid-Structure Interaction Problems in Arbitrary Lagrangian Eulerian Coordinates with the deal.II Library. IWR Report, University of Heidelberg, Germany, (2011).

[41] V. Chabannes, G. Pena, C. Prudhomme, High order fluid structure interaction in 2D and 3D Application to blood flow in arteries. Preprint submitted to J. Comput. Appl. Math., (2012). 